\title{
Hyperbolic Position Location Estimation in the Multipath Propagation Environment
}

\author{
Jacek Stefański \\ Gdansk University of Technology \\ 11/12 Narutowicza Str., PL-80233 Gdansk, Poland \\ jstef@eti.pg.gda.pl
}

\begin{abstract}
The efficiency analysis a hyperbolic position location estimation in the multipath propagation environment in the wideband code division multiple access (WCDMA) interface was presented. Four, the most popular methods: Chan's [1], Foy's [2], Fang's [3] and Friedlander's [4] were considered. These algorithms enable the calculation of the geographical position of a mobile station (MS) using the time differences of arrival (TDOA) between several base stations (BS) and MS. The simulation model is outlined and simulation results are presented.
\end{abstract}

\section{Introduction}

Hyperbolic position location estimation is accomplished in two stages. The first stage involves estimation of the time difference of arrival (TDOA) between transmitters through the use of time delay estimation techniques. The estimated TDOA's are then transformed into range difference measurements between base stations (BSs), resulting in a set of nonlinear hyperbolic equations. The second stage utilizes efficient algorithms to produce an unambiguous solution to these nonlinear hyperbolic equations.

Referring all TDOA's to the first base station, which is assumed to be the base station controlling, the distance between the $i$-th source (BS) and the receiver is given as

$$
R_{i}=\sqrt{\left(X_{i}-x\right)^{2}+\left(Y_{i}-y\right)^{2}}=\sqrt{X_{i}^{2}+Y_{i}^{2}-2 X_{i} x-2 Y_{i} y+x^{2}+y^{2}}
$$

where $\left(X_{i}, Y_{i}\right)$ and $(x, y)$ are coordination of $i$-th base station and mobile station (MS) respectively. The range difference between base stations with respect to the first arriving base station is

$$
R_{i, 1}=v \cdot t_{i, 1}=R_{i}-R_{1}=\sqrt{\left(X_{i}-x\right)^{2}+\left(Y_{i}-y\right)^{2}}-\sqrt{\left(X_{1}-x\right)^{2}+\left(Y_{1}-y\right)^{2}}
$$

where $v$ is the radio signal speed, $R_{i, I}$ is the range difference distance between the first base station and the $i$-th base station, $R_{I}$ is the distance between the first base station and the MS, and $t_{i, 1}$ is the estimated TDOA between the first base station and the $i$-th base station. This defines the set of nonlinear hyperbolic equations whose solution gives 2-D coordinates of the mobile station.

This paper presents the efficiency analysis a hyperbolic position location estimation methods in the multipath propagation environment, especially Chan's, Foy's, Fang's and Friedlander's algorithms. 


\section{Mathematical Model}

Solving the nonlinear equations of (2) is difficult. Consequently, linearizing this set of equations is commonly performed. One way of linearizing these equations is through the use of Taylor-series expansion and retaining the first two terms [2]. An commonly used alternative method to the Taylor-series expansion method, presented in [1], [3] and [4], is to first transform the set of nonlinear equations in (2) into another set of equations. Rearranging the form of (2) into

$$
R_{i}^{2}=\left(R_{i, 1}+R_{1}\right)^{2}
$$

Equation (1) can now be rewritten as

$$
R_{i, 1}^{2}+2 R_{i, 1} R_{1}+R_{1}^{2}=X_{i}^{2}+Y_{i}^{2}-2 X_{i} x-2 Y_{i} y+x^{2}+y^{2}
$$

Subtracting (1) at $i=1$ from (4) results in

$$
R_{i, 1}^{2}+2 R_{i, 1} R_{1}=X_{i}^{2}+Y_{i}^{2}-2 X_{i, 1} x-2 Y_{i, 1} y+X_{1}^{2}+Y_{1}^{2}
$$

where $X_{i, 1}$ and $Y_{i, 1}$ are equal to $X_{i}-X$ and $Y_{i}-Y$ respectively. The set of equations in (5) are now linear with the source location $(x, y)$ and the range of the first base station to the source $R_{l}$ as the unknowns, and are more easily handled.

In this paper a 2-D hyperbolic position location system using only three base station is considered.

\subsection{Chan's Method}

Following Chan's method [1], for a three base station system, producing two TDOA's, $x$ and $y$ can be solved in terms of $R_{l}$ from (5). The solution is in the form of

$$
\left[\begin{array}{l}
x \\
y
\end{array}\right]=-\left[\begin{array}{ll}
X_{2,1} & Y_{2,1} \\
X_{3,1} & Y_{3,1}
\end{array}\right]^{-1} \times\left\{\left[\begin{array}{l}
R_{2,1} \\
R_{3,1}
\end{array}\right] R_{1}+\frac{1}{2}\left[\begin{array}{c}
R_{2,1}^{2}-K_{2}+K_{1} \\
R_{3,1}^{2}-K_{3}+K_{1}
\end{array}\right]\right\}
$$

where $K_{1}=X_{1}^{2}+Y_{1}^{2}, K_{2}=X_{2}^{2}+Y_{2}^{2}, K_{3}=X_{3}^{2}+Y_{3}^{2}$. When (6) is inserted into (1), with $i=1$, a quadratic equation in terms of $R_{l}$ is produced

$$
a R_{1}^{2}+b R_{1}+c=0
$$

Substituting the positive root back into (6) results in the final solution. There may exist two positive roots from the quadratic equation that can produce two different solutions, resulting in an ambiguity. Simulations in this work have shown, that only the following root should be considered for cellular position location

$$
R_{1}=\frac{-b-\sqrt{b^{2}-4 a c}}{2 a}
$$

\subsection{Foy's Method}

The Foy's method linearizes the set of equations in (2) by Taylor-series expansion then uses an iterative method to solve the system of linear equations. The iterative 
method begins with an initial guess and improves the estimate at each iteration by determining the local linear least square solution. With a set of TDOA estimates, the method starts with an initial guess $\left(x_{0}, y_{0}\right)$ and computes the deviations $[\Delta x, \Delta y]^{T}$ of the position location estimation

$$
\left[\begin{array}{l}
\Delta x \\
\Delta y
\end{array}\right]=\left(\mathbf{G}^{\mathbf{T}} \cdot \mathbf{G}\right)^{-1} \cdot \mathbf{G}^{\mathbf{T}} \cdot \mathbf{h}
$$

where

$$
\begin{gathered}
\mathbf{h}=\left[\begin{array}{l}
R_{2,1}-\left(R_{2}-R_{1}\right) \\
R_{3,1}-\left(R_{3}-R_{1}\right)
\end{array}\right] \\
\mathbf{G}=\left[\begin{array}{ll}
\frac{X_{1}-x}{R_{1}}-\frac{X_{2}-x}{R_{2}} & \frac{Y_{1}-y}{R_{1}}-\frac{Y_{2}-y}{R_{2}} \\
\frac{X_{1}-x}{R_{1}}-\frac{X_{3}-x}{R_{3}} & \frac{Y_{1}-y}{R_{1}}-\frac{Y_{3}-y}{R_{3}}
\end{array}\right]
\end{gathered}
$$

The values $R_{1}$ and $R_{2}$ are computed from (1) with $x=x_{0}$ and $y=y_{0}$. In the next iteration, $x_{0}$ and $y_{0}$-are set to $x_{0}+\Delta x$ and $y_{0}+\Delta y$. The whole process is repeated until $\Delta x$ and $\Delta y$ are sufficiently small, resulting in the estimated position location of the $(x, y)$. This Taylor-series method can provide accurate results, however, it requires a close initial guess $\left(x_{0}, y_{0}\right)$ to guarantee convergence and can be very computationally intensive.

\subsection{Fang's Method}

Fang establishes a coordinate system so that the first base station is located at $(0,0)$, the second $\mathrm{BS}$ at $\left(X_{2}, 0\right)$ and the third $\mathrm{BS}$ at $\left(X_{3}, Y_{3}\right)$. The following relationships are simplified

$$
\begin{gathered}
R_{i}=\sqrt{\left(X_{i}-x\right)^{2}+\left(Y_{i}-y\right)^{2}}=\sqrt{x^{2}+y^{2}} \\
X_{i, 1}=X_{i}-X_{1}=X_{i} \\
Y_{i, 1}=Y_{i}-Y_{1}=Y_{i}
\end{gathered}
$$

Using these relationships, the equation of (5) can be rewritten as

$$
\begin{gathered}
2 R_{2,1} R_{1}=-R_{2,1}^{2}+X_{2}^{2}-2 X_{2} x \\
2 R_{3,1} R_{1}=-R_{3,1}^{2}+X_{3}^{2}+Y_{3}^{2}-2 X_{3} x-2 Y_{3} y
\end{gathered}
$$

Equating the two equations and simplifying results in

$$
y=g \cdot x+h
$$


where

$$
\begin{gathered}
g=\left\{R_{3,1} \cdot\left(X_{2} / R_{2,1}\right)-X_{3}\right\} / Y_{3} \\
h=\left\{X_{3}^{2}+Y_{3}^{2}-R_{3,1}^{2}+R_{3,1} \cdot R_{2,1} \cdot\left(1-\left(X_{2} / R_{2,1}\right)^{2}\right)\right\} / 2 \cdot Y_{3}
\end{gathered}
$$

Substituting equation (17) into the equation (15) results in

$$
d \cdot x^{2}+e \cdot x+f=0
$$

where

$$
\begin{gathered}
d=-\left\{1-\left(X_{2} / R_{2,1}\right)^{2}+g^{2}\right\} \\
e=X_{2} \cdot\left\{\left(1-\left(X_{2} / R_{2,1}\right)^{2}\right\}-2 g h\right. \\
f=\left(R_{2,1}^{2} / 4\right) \cdot\left\{1-\left(X_{2} / R_{2,1}\right)^{2}\right\}^{2}-h^{2}
\end{gathered}
$$

Solving the quadratic equation (20), we get two values for $x$. Using a priori information, one of the values is chosen and is used to find out $y$ from (17). It has been found by simulations in this research that one of the roots of (20) is beyond the cell coverage area. Hence for position location in cellular systems we only need to evaluate the following root from (20)

$$
x=\frac{-e-\sqrt{e^{2}-4 d f}}{2 d}
$$

As stated earlier, putting this value of $x$ in (17) will give us the other coordinate of the mobile's position estimate.

\subsection{Friedlander's Method}

Friedlander's method [4] utilizes a least squares error criterion to solve for the position location. He first transforms the linear set of equations of (5) into

$$
X_{i, 1} x+Y_{i, 1} y=0.5 \cdot\left(X_{i}^{2}+Y_{i}^{2}-X_{1}^{2}-Y_{1}^{2}-R_{i, 1}^{2}\right)-R_{i, 1} R_{1}
$$

Then realizes this equation in matrix form as

$$
\mathbf{S} \cdot \mathbf{X}=\mathbf{u}-R_{1} \cdot \mathbf{p}
$$

where

$$
\begin{gathered}
\mathbf{S}=\left[\begin{array}{ll}
X_{2,1} & Y_{2,1} \\
X_{3,1} & Y_{3,1}
\end{array}\right] \\
\mathbf{x}=\left[\begin{array}{ll}
x & y
\end{array}\right]^{T}
\end{gathered}
$$




$$
\begin{gathered}
\mathbf{u}=\left[\begin{array}{c}
X_{2}^{2}+Y_{2}^{2}-X_{1}^{2}-Y_{1}^{2}-R_{2,1}^{2} \\
X_{3}^{2}+Y_{3}^{2}-X_{1}^{2}-Y_{1}^{2}-R_{3,1}^{2}
\end{array}\right] \\
\mathbf{p}=\left[\begin{array}{ll}
R_{2,1} & R_{3,1}
\end{array}\right]^{T}
\end{gathered}
$$

In order to eliminate the second term of (26), which requires knowledge of the unknown term $R_{l}$, the equation in (26) is premultiplied by a matrix $\mathbf{N}$ which has $\mathbf{p}$ in its null-space. Matrix $\mathbf{N}$ is defined as

$$
\mathbf{N}=(\mathbf{I}-\mathbf{Z}) \cdot \mathbf{D}
$$

where

$$
\begin{gathered}
\mathbf{D}=(\operatorname{diag}\{\mathbf{p}\})^{-1}=\left[\begin{array}{cc}
R_{2,1} & 0 \\
0 & R_{3,1}
\end{array}\right]^{-1} \\
\mathbf{Z}=\left[\begin{array}{ll}
0 & 1 \\
1 & 0
\end{array}\right], \quad \mathbf{I}=\left[\begin{array}{ll}
1 & 0 \\
0 & 1
\end{array}\right] .
\end{gathered}
$$

Closed form solution for the coordinates of the source is found by solving

$$
\mathbf{N} \cdot \mathbf{S} \cdot \mathbf{x}=\mathbf{N} \cdot \mathbf{u}
$$

The mobile station position can then be computed using the least square solution. A closed form solution which can be used is given by Friedlander as

$$
\mathbf{X}=\left(\mathbf{S}^{\mathbf{T}} \cdot \mathbf{N}^{\mathbf{T}} \cdot \mathbf{N} \cdot \mathbf{S}\right)^{-1} \cdot \mathbf{S}^{\mathbf{T}} \cdot \mathbf{N}^{\mathbf{T}} \cdot \mathbf{N} \cdot \mathbf{u}
$$

\section{Simulation Model}

The experiments were carried out using the simulation model required for the UMTS [6]. This is a typical bad urban environmental model (Manhattan model). The area consisted 32 blocks with a total number of 21 base stations. The street width was 30 $\mathrm{m}$ and the distance between two street corners was $230 \mathrm{~m}$ (see Fig. 1). Base station antennas were placed $10 \mathrm{~m}$ above the mobile users but below rooftops. In our implementation, we covered the simulation area with a regular grid with resolution of $10 \mathrm{~m}$. In the simulation model, the effect of a multipath propagation was implemented.

The time of radio signal arrival between the mobile station and base stations under the multipath environment was modeled by the sum of true value $\tau_{0}$ and non-line of sight (NLOS) error $\tau_{m}$ [7]

$$
\tau=\tau_{0}+\tau_{m}
$$

The variable $\tau_{m}$ is defined as mean excess delay and essentially correlated with the root mean squared delay spread $\tau_{\text {rms }}$ [8] 


$$
\tau_{m} \approx k \cdot \tau_{r m s}=k \cdot T_{1} \cdot d^{\varepsilon} \cdot y
$$

where $k$ is a constant proportional coefficient ( $k=1$ in urban region), $T_{l}$ is the median value of $\tau_{r m s}$ at $d=1 \mathrm{~km}$ (for urban environment $T_{l}=0.7 \mu \mathrm{s}$ ), $d$ is the distance between the mobile station and base station, $\varepsilon$ is an exponent (for urban environment $\varepsilon=0.5$ ) and $y$ is a lognormal variate. Specifically,

$$
Y=10 \cdot \lg y
$$

is a Gaussian random variable over the terrain at the distance $d$, having zero mean and a standard deviation $\sigma_{\mathrm{y}}$ (for urban environment $\sigma_{\mathrm{y}}=4 \mathrm{~dB}$ ).

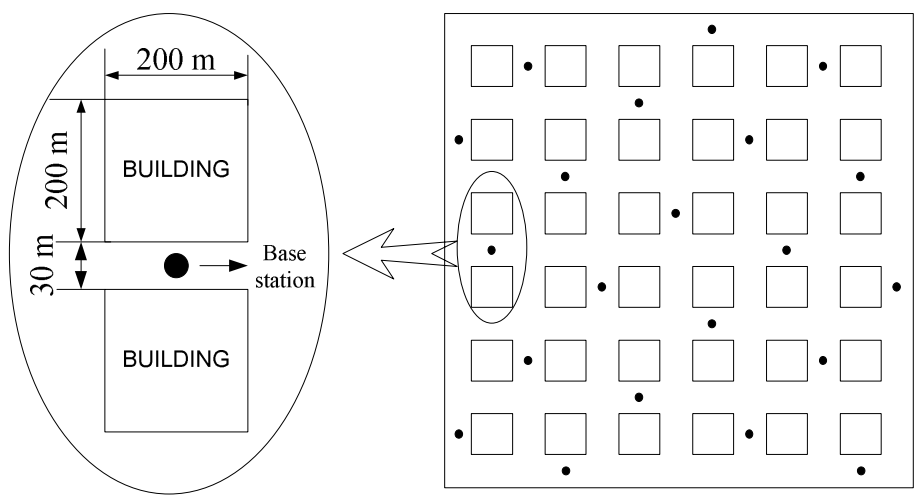

Fig. 1. Simulation model of the bad urban environment (Manhattan model)

\section{Simulation Results}

The most commonly used measure of positioning accuracy is the root mean square error metric. For a one dimensional case its value can be calculated as in [5]

$$
R M S_{x}=\sqrt{\frac{\sum_{i=1}^{n}\left(x_{i}-x_{t}\right)^{2}}{n}}, R M S_{y}=\sqrt{\frac{\sum_{i=1}^{n}\left(y_{i}-y_{t}\right)^{2}}{n}}
$$

where $x_{t}$ and $y_{t}$ denote the true MS coordinate, $x_{i}$ and $y_{i}$ its estimation, and $n$ $(n=10000)$ represents a number of measurements. For the purpose of this article, however, the two dimensional metric is exploited instead. It can be easily derived by calculating the one dimensional metric for both coordinates, and than final value can be computed

$$
R M S_{x y}=\sqrt{R M S_{x}^{2}+R M S_{y}^{2}} .
$$

Such a measure is very useful due to a possibility of representing it on a plane as a circle, which a center is the true target's position and radius' length is $R M S_{x y}$ value. Within such a circle there are about $63 \%$ target's position estimations. Basing on the 
two dimensional root mean square error metric, another circle can be created. Its radius' length is two times longer than the previous one's. Within this circle there are about $98 \%$ target's position estimations. Moreover, the cumulative probability distribution functions (CDF) of the absolute position error were obtained from the simulation investigations. The absolute position error is defined as

$$
\Delta d=\sqrt{\left(x-x_{t}\right)^{2}+\left(y-y_{t}\right)^{2}}
$$

where $x$ and $y$ denote the estimated coordinates of a mobile station. All timing values have been assumed to be accurate within $\pm 1 / 2$ of a WCDMA chip (the uniform random time error corresponds to a maximum distance error of about $\pm 38 \mathrm{~m}$ ).

Comparisons of errors for the different methods are in Tab. 1. The simulation results are presented in Fig. 2. The hyperbolic position location estimation methods presented offer different accuracy's and complexities. The Chan's method offers a closed form solution, thus eliminating the need for an iteration approach, but requires a priori information to eliminate ambiguities. The Foy's method, using Taylor-series least square method offers accurate position location estimation at reasonable noise levels and is applicable to any number of range difference measurements, but can be computational intensive.

Table 1. Comparison of errors for the different methods

\begin{tabular}{|l|c|c|c|}
\hline \multirow{2}{*}{ Method } & \multicolumn{3}{|c|}{ Type of errors } \\
\cline { 2 - 4 } & $\boldsymbol{R M S}_{\boldsymbol{x}}[\mathbf{m}]$ & $\boldsymbol{R} \boldsymbol{M} \boldsymbol{S}_{\boldsymbol{y}}[\mathbf{m}]$ & $\boldsymbol{R M S}_{\boldsymbol{x y}}[\mathbf{m}]$ \\
\hline Chan & 82.13 & 134.05 & 157.21 \\
\hline Foy & 107.99 & 103.60 & 149.65 \\
\hline Fang & 78.19 & 108.61 & 133.83 \\
\hline Friedlander & 240.79 & 233.35 & 335.31 \\
\hline
\end{tabular}

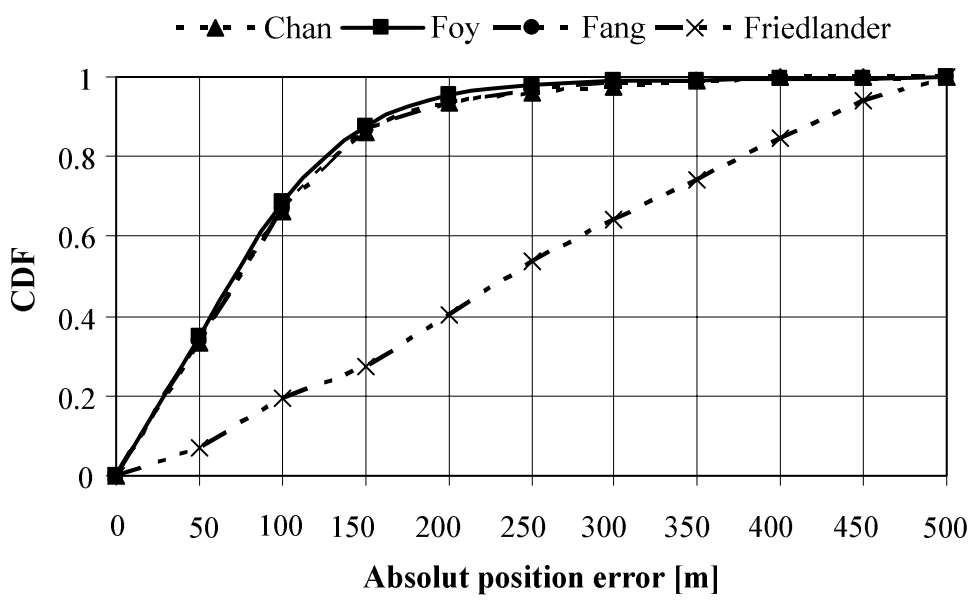

Fig. 2. The CDFs of absolute position error for the proposed methods 
The Taylor-series method is iterative and has the risk of convergence to local minima. The Fang's method provides an optimal solution when the system of equations is consistent but does not make use of redundant measurements (only tree base stations are needed). The Friedlander's approach reduces the computational requirements for the solution but does is suboptimal because it eliminates a fundamental relationship and is difficult to implementation - inverse matrix with very low or high values.

\section{Summary}

To summarize Chan's method offers a closed form and is the best available option for solving hyperbolic equations (2). This method also requires a priori knowledge of the approximate location and distance between mobile station and first (serving) base station.

The optimal position location algorithm for a given situation depends on the geometrical configuration of the base stations, the number of coordinates of the source to be solved and range difference measurements utilized, computational requirements and complexity, assumptions on the statistical nature of the channel and desired accuracy.

An interesting observation that was made while studying the ambiguities in the Fang's and Chan's algorithms was that both these ambiguities are essentially the same. It was seen that if we make wrong choices in both algorithms for a given case then the wrong results given by both algorithms are identical.

Acknowledgments. This work was supported by the Ministry of Education and Science of the Republic of Poland, under grant No. O R00 004906.

\section{References}

[1] Chan, Y.T., Ho, K.C.: A Simple and Efficient Estimator for Hyperbolic Location. IEEE Trans. on Signal Proc. 42(8), 1905-1915 (1994)

[2] Foy, W.H.: Position-Location Solutions by Taylor-Series Estimation. IEEE Trans. on Aero. and Elec. Systems AES-12(2), 187-194 (1976)

[3] Fang, B.T.: Simple Solution for Hyperbolic and Related Position Fixes. IEEE Trans. on Aero. and Elec. Systems 26(5), 748-753 (1990)

[4] Friedlander, B.: A Passive Localization Algorithm and Its Accuracy Analysis. IEEE Jour. of Oceanic Engineer. OE-12(1), 234-245 (1987)

[5] Bronk, K., Stefanski, J.: Bad Geometry Effect in TDOA Systems. Polish Journal of Environmental Studies 16(4B), 11-13 (2007)

[6] ETSI, Universal Mobile Telecommunications System (UMTS); Selection procedures for the choice of radio transmission technologies of the UMTS, TR 101 112, ver. 3.2.0. (April 1998)

[7] Stefanski, J.: Method of Location of a Mobile Station in the WCDMA System without Knowledge of Relative Time Differences. In: Proc. of IEEE 65th Vehicular Technology Conference, pp. 674-678 (2007)

[8] Greenstain, L.J., Erceg, V., Yeh, Y.S., Clark, M.V.: A new path-gain/delay-spread propagation model for digital cellular channels. IEEE Trans. on Vehicular Techn. 46, 477-485 (1997) 